\title{
The distributions of sums for Shanker, Akash, Ishita, Pranav, Rani, and Ram
}

\author{
Awadh \\ Afshin Yaghoubi \\ Department of Industrial Engineering, Sharif University of Technology, Tehran, Iran; \\ Email: afshin.yaghoubi@alum.sharif.edu
}

\begin{abstract}
In statistics and probability theory, one of the most important statistics is the sums of random variables. After introducing a probability distribution, determining the sum of $n$ independent and identically distributed random variables is one of the interesting topics for authors. This paper presents the probability density functions for the sum of $n$ independent and identically distributed random variables such as Shanker, Akash, Ishita, Pranav, Rani, and Ram Awadh. In order to determine all aforementioned distributions, the problem-solving methods are applied which is based on the change-of-variables technique.
\end{abstract}

Keywords: Akash distribution, Ishita distribution, Pranav distribution, Ram Awadh distribution, Rani distribution, Shanker distribution.

\section{Introduction}

The items such as various wireless communications (e.g., satellite communications, equal-gain receivers, and radars) and insurance are widely known as applications for the sums of random variables (Karagiannidis et al. (2005), Van Khuong and Kong, (2006), Nadarajah (2008), Ramsay (2008), and Nadarajah et al. (2012)). Moreover, in the field of systems reliability, like the lifespan of 1 out of $n$ cold standby, spare systems can also be referred to the sums of random variables. If $S_{n}$ represents the lifetime of the whole system and $X_{i}$ indicates the lifetime of the $i$ th component, then the sums of $X_{i}$ 's lifetime is equal to $S_{n}$.

Recently, a collection of the one-parameter continuous probability density functions (PDFs) has been introduced by Shukla and Shanker (2019). Similar to the Lindley distribution, these functions are also made up of a mixture of exponential distribution with scale parameter $\theta$, $(\exp (\theta))$ and gamma distribution with different shape parameters $n$, and scale parameters $\theta$, $\operatorname{(gamma}(n, \theta))$. For instance, with $n=2$ one can obtain the Shanker distribution. When $n=3$, the 
Akash and the Ishita distributions are derived. For $n=4,5$ and 6, the Pranav, Rani and Ram Awadh distributions are obtainable, respectively.

Let consider $f_{E}(x)$ and $f_{G}(x)$ as the exponential and the gamma PDFs, respectively. In this case, their combination with a specified ratio of $p$, namely $f(x)=p f_{E}(x)+(1-p) f_{G}(x)$ represents the proposed PDFs. The information required to create $f(x)$ 's are summarized in Table 1.

Table 1. Information of $f(x)$

\begin{tabular}{cccc}
\hline PDFs & $\boldsymbol{f}_{\boldsymbol{E}}(\boldsymbol{x})$ & $\boldsymbol{f}_{\boldsymbol{G}}(\boldsymbol{x})$ & Proportions, $\boldsymbol{p}$ \\
\hline Shanker & $\theta e^{-\theta x}$ & $\theta^{2} x e^{-\theta x}$ & $\theta^{2} / \theta^{2}+1$ \\
Akash & $\theta e^{-\theta x}$ & $\theta^{3} x^{2} e^{-\theta x} / 2 !$ & $\theta^{2} / \theta^{2}+2$ \\
Ishita & $\theta e^{-\theta x}$ & $\theta^{3} x^{2} e^{-\theta x} / 2 !$ & $\theta^{3} / \theta^{3}+2$ \\
Pranav & $\theta e^{-\theta x}$ & $\theta^{4} x^{3} e^{-\theta x} / 3 !$ & $\theta^{4} / \theta^{4}+6$ \\
Rani & $\theta e^{-\theta x}$ & $\theta^{5} x^{4} e^{-\theta x} / 4 !$ & $\theta^{5} / \theta^{5}+24$ \\
Ram Awadh & $\theta e^{-\theta x}$ & $\theta^{6} x^{5} e^{-\theta x} / 5 !$ & $\theta^{6} / \theta^{6}+120$ \\
\hline
\end{tabular}

Using Table 1 data, the presented PDFs by Shukla and Shanker (2019) are given in Table 2.

Table 2. The PDFs introduced

\begin{tabular}{ccc}
\hline Distributions & \multicolumn{1}{c}{ PDFs } & By \\
\hline Shanker & $f(x)=\frac{\theta^{2}}{\theta^{2}+1}(\theta+x) e^{-\theta x}$ & Shanker, 2015 a \\
Akash & $f(x)=\frac{\theta^{3}}{\theta^{2}+2}\left(1+x^{2}\right) e^{-\theta x}$ & Shanker, 2015 b \\
Ishita & $f(x)=\frac{\theta^{3}}{\theta^{3}+2}\left(\theta+x^{2}\right) e^{-\theta x}$ & Shanker and Shukla, 2017 \\
Pranav & $f(x)=\frac{\theta^{4}}{\theta^{4}+6}\left(\theta+x^{3}\right) e^{-\theta x}$ & Shukla, 2018 \\
Rani & $f(x)=\frac{\theta^{5}}{\theta^{5}+24}\left(\theta+x^{4}\right) e^{-\theta x}$ & Shanker, 2017 \\
Ram Awadh & $f(x)=\frac{\theta^{6}}{\theta^{6}+120}\left(\theta+x^{5}\right) e^{-\theta x}$ & Shukla, 2018 \\
\hline
\end{tabular}

The authors have compared available distributions in Table 2 with the Lindley distribution and the results showed that the performance of the them is better than the Lindley distribution.

The Lindley distribution was introduced by Lindley (1958). This distribution is plentifully used in the reliability theory. Ghitany et al. (2008) examined the Lindley distribution in detail and showed that this distribution in many ways has more flexibility than exponential distribution.

Introducers of the presented PDFs in Table 2 have discussed some features such as descriptive statistics (mean, variance, coefficient of variation, skewness and kurtosis), reliability theory (hazard function, mean residual life and stress-strength reliability models), stochastic ordering, mean deviations, Lorenz curves, entropies and simulation study. But another important aspect, 
i.e., the sum of $n$ independent and identically distributed (IID) random variables for introduced functions, has not been studied. Therefore, here we calculate the distribution of sums for the aforementioned PDFs.

The prevalent and well-known methods for obtaining the sums of $n$ IID random variables are the moment generating functions, Laplace transforms, convolution method, and change-of-variables technique. These methods can be used to find the sum of the distributions which mathematically have a simple PDF (e.g., exponential, gamma, Lindley, and Pareto). However, the other density functions like the Weibull and Burr that have a more complex mathematical form, to obtain the distribution of sums are used methods of the generalized hypergeometric functions such as the Fox's H-function and the Meijer's G-function.

There is an extended history for the sums of independent random variables. Many authors have examined the distribution of sums of the random variables with a statistical distribution. Some of the works devoted to the sum of independent random variables can be seen briefly in Table 3 .

Table 3. Pertinent literature on the distribution of sums

\begin{tabular}{|c|c|c|}
\hline $\begin{array}{l}\text { Type of distribution of } \\
\text { sums }\end{array}$ & Authors & Solving methods \\
\hline Exponential & $\begin{array}{l}\text { Amari and Misra } \\
\text { (1997) }\end{array}$ & Laplace transform \\
\hline Exponential & $\begin{array}{l}\text { Van Khuong and Kong } \\
\text { (2006) }\end{array}$ & Characteristic function \\
\hline Lindley & $\begin{array}{l}\text { Zakerzadeh and Dolati } \\
\text { (2009) }\end{array}$ & Moment generating function \\
\hline Lindley & $\begin{array}{l}\text { Hassan } \\
(2014)\end{array}$ & Convolution \\
\hline Mid truncated Lindley & $\begin{array}{l}\text { El-Din et al. } \\
\text { (2010) }\end{array}$ & Laplace transform \\
\hline Pareto & $\begin{array}{c}\text { Ramsey } \\
(2008)\end{array}$ & Laplace transform \\
\hline Gamma & $\begin{array}{l}\text { Mathai } \\
(1982)\end{array}$ & Moment generating function \\
\hline Gamma & $\begin{array}{l}\text { Moschopoulos } \\
\text { (1984) }\end{array}$ & Moment generating function \\
\hline Rayleigh & $\begin{array}{l}\text { Karagiannidis et al. } \\
\qquad(2005)\end{array}$ & $\begin{array}{c}\text { Meijer's and generalized Hypergeometric } \\
\text { functions }\end{array}$ \\
\hline Weibull & $\begin{array}{l}\text { Yacoub } \\
(2006)\end{array}$ & Generalized gamma distribution \\
\hline Weibull & $\begin{array}{c}\text { Bouanani and Costa } \\
\text { (2018) }\end{array}$ & Meijer's G-function \\
\hline Burr (types III and XII) & $\begin{array}{l}\text { Nadarajah et al. } \\
\text { (2012) }\end{array}$ & $\begin{array}{l}\text { Fox's H-function, and Wright generalized } \Psi \text { - } \\
\text { function }\end{array}$ \\
\hline
\end{tabular}




\section{The distribution of sums}

Suppose $X_{1}, X_{2}, \ldots, X_{n}$ denote $n$ IID random variables that according to the PDFs of Table 2. If $S_{n}=X_{1}+X_{2}+\cdots+X_{n}$ is the distribution of sums for the PDFs listed in Table 2, by applying the change-of-variables technique one can obtain the density functions of $S_{n}$ for Shanker, Akash, Ishita, Pranav, Rani, and Ram Awadh. The results are briefly shown in Table 4.

Table 4. Distribution of sums for PDFs of Table 2

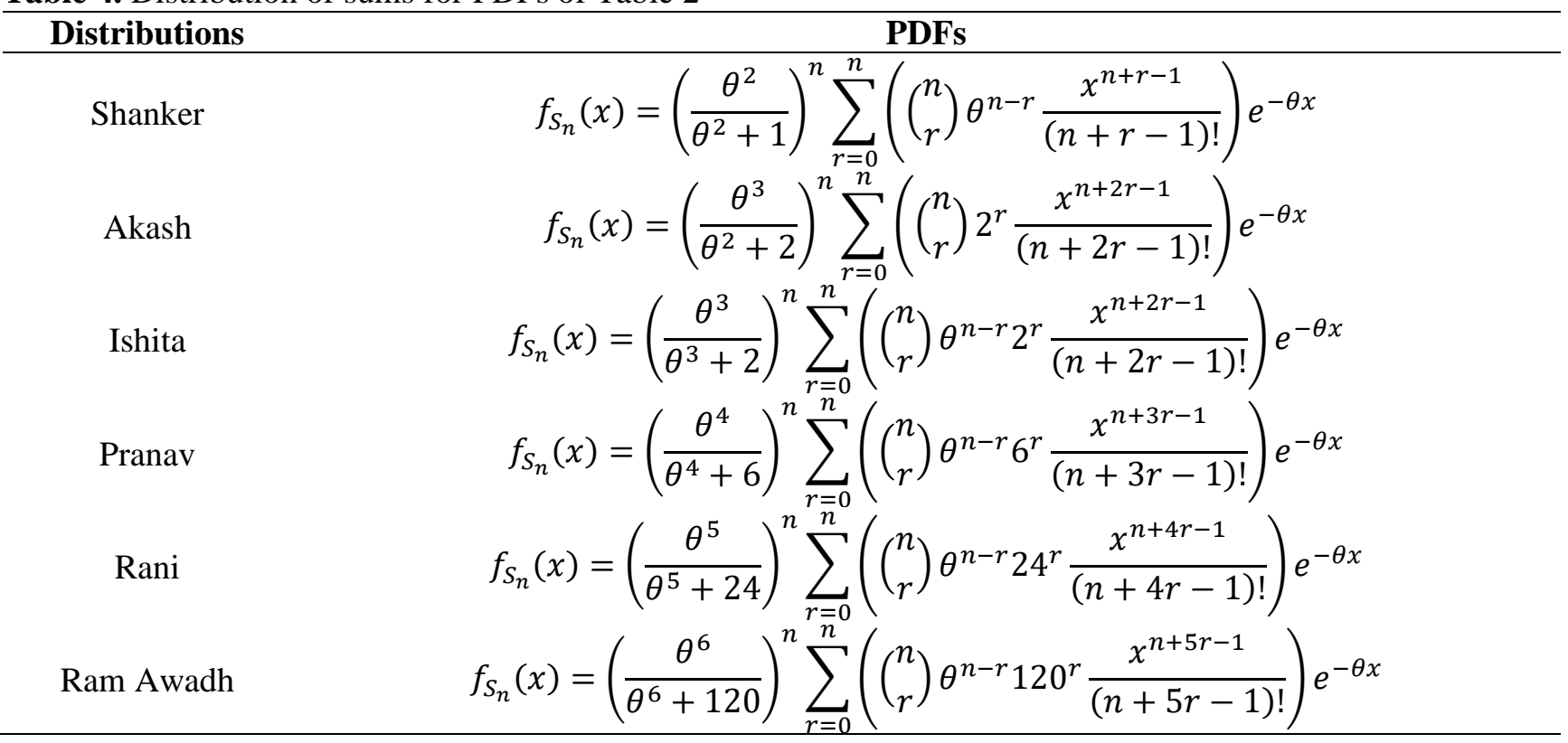

It can be simply shown that all the functions provided in Table 4 are PDF. For this purpose, it must be proved that $\int_{0}^{\infty} f_{S_{n}}(x) d x=1$.

In order to prove the PDFs of Table 4, we arbitrarily select the Ishita distribution.

Assuming $X_{i}=Y_{i}, i=1,2, \ldots, n-1$ and $X_{n}=S_{n}-\sum_{i=1}^{n-1} Y_{i}$, we the have:

$$
f_{Y_{1}, Y_{2}, \ldots, S_{n}}\left(y_{1}, y_{2}, \ldots, s_{n}\right)=f_{X_{1}, X_{2}, \ldots, X_{n}}\left(y_{1}, y_{2}, \ldots, s_{n}-\sum_{i=1}^{n-1} y_{i}\right) \cdot|J|
$$

where, $|J|$ is the absolute value of the Jacobian.

As the relationship between $X_{i}$ and $Y_{i}$ is linear, so $|J|=\left|\frac{\partial\left(X_{1}, X_{2}, \ldots, X_{n}\right)}{\partial\left(Y_{1}, Y_{2}, \ldots, S_{n}\right)}\right|=1$.

Since $X_{i}$ is independent, hence Eq. (1) is given by:

$$
f_{Y_{1}, Y_{2}, \ldots, S_{n}}\left(y_{1}, y_{2}, \ldots, s_{n}\right)=f_{X_{1}}\left(y_{1}\right) f_{X_{2}}\left(y_{2}\right) \ldots f_{X_{n}}\left(s_{n}-\sum_{i=1}^{n-1} y_{i}\right) ; y_{i}>0, s_{n}>\sum_{i=1}^{n-1} y_{i}
$$


Now, the marginal PDF for $S_{n}$ is equal to:

$$
\begin{array}{r}
f_{S_{n}}(x)=\left(\frac{\theta^{3}}{\theta^{3}+2}\right)^{n} e^{-\theta x} \int_{0}^{x} \int_{0}^{x-y_{1}} \ldots \int_{0}^{x-\sum_{i=1}^{n-2} y_{i}}\left(\prod_{i=1}^{n-1}\left(\theta+y_{i}^{2}\right)\right) \\
\left(\theta+\left(x-\sum_{i=1}^{n-1} y_{i}^{2}\right)^{2}\right) d y_{n-1} \ldots d y_{2} d y_{1}
\end{array}
$$

With $n=2$ and 3 in Eq. (3) and for $x>0$, the functions of $f_{S_{n}}(x)$ are given by:

$$
\begin{gathered}
f_{S_{2}}(x)=\left(\frac{\theta^{3}}{\theta^{2}+2}\right)^{2} e^{-\theta x} \int_{0}^{x}\left(\theta+y_{1}^{2}\right)\left(\theta+(x-y)^{2}\right) d y_{1} \\
=\left(\frac{\theta^{3}}{\theta^{3}+2}\right)^{2} e^{-\theta x}\left(x \theta^{2}+\frac{2}{3} \theta x^{3}+\frac{x^{5}}{30}\right)=\left(\frac{\theta^{3}}{\theta^{3}+2}\right)^{2} e^{-\theta x}\left(\theta^{2} \frac{x^{1}}{1 !}+4 \theta \frac{x^{3}}{3 !}+4 \frac{x^{5}}{5 !}\right)
\end{gathered}
$$

In Eq. (4), it can easily be observed that the coefficients of $\frac{x^{i}}{i !}$ in the expression of $\left(\theta^{2} \frac{x^{1}}{1 !}+\right.$ $\left.4 \theta \frac{x^{3}}{3 !}+4 \frac{x^{5}}{5 !}\right)$ is equal to $(\theta+2)^{2}$.

For $S_{3}$, we have:

$$
\begin{gathered}
f_{S_{3}}(x)=\left(\frac{\theta^{3}}{\theta^{3}+2}\right)^{3} e^{-\theta x} \int_{0}^{x} \int_{0}^{x-y_{1}}\left(\theta+y_{1}^{2}\right)\left(\theta+y_{2}^{2}\right)\left(\theta+\left(x-y_{1}-y_{2}\right)^{2}\right) d y_{2} d y_{1} \\
=\left(\frac{\theta^{3}}{\theta^{3}+2}\right)^{3} e^{-\theta x}\left(\frac{x^{2}}{2} \theta^{3}+\frac{x^{4}}{4} \theta^{2}+\frac{x^{6}}{60} \theta+\frac{x^{8}}{5040}\right) \\
=\left(\frac{\theta^{3}}{\theta^{3}+2}\right)^{3} e^{-\theta x}\left(\theta^{3} \frac{x^{2}}{2 !}+6 \theta^{2} \frac{x^{4}}{4 !}+12 \theta \frac{x^{6}}{6 !}+8 \frac{x^{8}}{8 !}\right)
\end{gathered}
$$

Similarly, the coefficients of $\frac{x^{i}}{i !}$ in the phrase of $\left(\theta^{3} \frac{x^{2}}{2 !}+6 \theta^{2} \frac{x^{4}}{4 !}+12 \theta \frac{x^{6}}{6 !}+8 \frac{x^{8}}{8 !}\right)$ are equal to $(\theta+2)^{3}$. Therefore, it can be said that the coefficients of $\frac{x^{i}}{i !}$ for the sum of $n$ Ishita random variables are $(\theta+2)^{n}$. Nevertheless, according to the obtained results of Eqs. (4) and (5), the explicit form for the sum of $n$ IID with Ishita random variables can be expressed as follows:

$$
f_{S_{n}}(x)=\left(\frac{\theta^{3}}{\theta^{3}+2}\right)^{n}\left(\sum_{r=0}^{n}\left(\begin{array}{l}
n \\
r
\end{array}\right) 2^{r} \theta^{n-r} \frac{x^{n+2 r-1}}{(n+2 r-1) !}\right) e^{-\theta x}
$$

By this analogous logic, it can be simplicity obtained all the PDFs of $S_{n}$ reported in Table 4.

\section{The $m$ th Moments}


Another important measure for statistical distributions is the $m$ th moment. The calculation of this factor is very important for any statistical distribution. The $m$ th moment for each arbitrary PDF can be expressed as:

$$
E\left(X^{m}\right)=\int_{0}^{\infty} x^{m} f_{S_{n}}(x) d x
$$

By special values for amounts of $m$ can be defined the descriptive statistical indicators such as, mean, variance, coefficient of variation, skewness and kurtosis. Table 5 presents the closed form for the $m$ th moments of PDFs Table 4 as follows.

Table 5. The $m$ th moments for PDFs of Table 3

\begin{tabular}{cc}
\hline Distributions & $\frac{m !}{\theta^{m}}\left(\frac{\theta^{2}}{\theta^{2}+1}\right)^{n} \sum_{r=0}^{n}\left(\begin{array}{l}n \\
r\end{array}\right)\left(\begin{array}{c}n+m+r-1 \\
n+r-1\end{array}\right)\left(\frac{1}{\theta^{2}}\right)^{r}$ \\
\hline Akash & $\frac{m !}{\theta^{m}}\left(\frac{\theta^{2}}{\theta^{2}+2}\right)^{n} \sum_{r=0}^{n}\left(\begin{array}{l}n \\
r\end{array}\right)\left(\begin{array}{c}n+m+2 r-1 \\
n+2 r-1\end{array}\right)\left(\frac{2}{\theta^{2}}\right)^{r}$ \\
Ishita & $\frac{m !}{\theta^{m}}\left(\frac{\theta^{3}}{\theta^{3}+2}\right)^{n} \sum_{r=0}^{n}\left(\begin{array}{l}n \\
r\end{array}\right)\left(\begin{array}{c}n+m+2 r-1 \\
n+2 r-1\end{array}\right)\left(\frac{2}{\theta^{3}}\right)^{r}$ \\
Pranav & $\frac{m !}{\theta^{m}}\left(\frac{\theta^{4}}{\theta^{4}+6}\right)^{n} \sum_{r=0}^{n}\left(\begin{array}{l}n \\
r\end{array}\right)\left(\begin{array}{c}n+m+3 r-1 \\
n+3 r-1\end{array}\right)\left(\frac{6}{\theta^{4}}\right)^{r}$ \\
Rani & $\frac{m !}{\theta^{m}}\left(\frac{\theta^{5}}{\theta^{5}+24}\right)^{n} \sum_{r=0}^{n}\left(\begin{array}{l}n \\
r\end{array}\right)\left(\begin{array}{c}n+m+4 r-1 \\
n+4 r-1\end{array}\right)\left(\frac{24}{\theta^{5}}\right)^{r}$ \\
Ram Awadh & $\frac{m !}{\theta^{m}}\left(\frac{\theta^{6}}{\theta^{6}+120}\right)^{n} \sum_{r=0}^{n}\left(\begin{array}{l}n \\
r\end{array}\right)\left(\begin{array}{c}n+m+5 r-1 \\
n+5 r-1\end{array}\right)\left(\frac{120}{\theta^{6}}\right)^{r}$ \\
\hline Contusion &
\end{tabular}

\section{Conclusion}

In this paper, the probability density functions of the sum of $n$ independent and identically distributed random variables with Shanker, Akash, Ishita, Pranav, Rani, and Ram Awadh have been explicitly assessed. Their determination is based on the change-of-variables technique. Moreover, the $m$ th moments for them were also accurately calculated.

\section{References}

Amari, S. V., \& Misra, R. B. (1997). Closed-form expressions for distribution of sum of exponential random variables. IEEE Transactions on reliability, 46(4), 519-522.

El Bouanani, F., \& da Costa, D. B. (2018). Accurate closed-form approximations for the sum of correlated Weibull random variables. IEEE Wireless Communications Letters, 7(4), 498-501. 
El-Din, M., Teamah, A. A. M., Salem, A. M., \& Abd El-Bar, A. M. (2010). Random sum of mid truncated Lindley distribution. J. Adv. Res. Stat. Probab, 2(1), 27-36.

Ghitany, M. E., Atieh, B., \& Nadarajah, S. (2008). Lindley distribution and its application. Mathematics and computers in simulation, 78(4), 493-506.

Hassan, M. K. (2014). On the convolution of Lindley distribution. Columbia In ternational Publishing Contemporary Mathematics and Statistics, 2(1), 47-54.

Karagiannidis, G. K., Tsiftsis, T. A., \& Sagias, N. C. (2005). A closed-form upper-bound for the distribution of the weighted sum of Rayleigh variates. IEEE Communications Letters, 9(7), 589591.

Lindley, D. V. (1958). Fiducial distributions and Bayes' theorem. Journal of the Royal Statistical Society. Series B (Methodological), 102-107.

Mathai, A. M. (1982). Storage capacity of a dam with gamma type inputs. Annals of the Institute of Statistical Mathematics, 34(3), 591-597.

Moschopoulos, P. G. (1985). The distribution of the sum of independent gamma random variables. Annals of the Institute of Statistical Mathematics, 37(3), 541-544.

Nadarajah, S., Pogány, T. K., \& Saxena, R. K. (2012). On the characteristic function for Burr distributions. Statistics, 46(3), 419-428.

Nadarajah, S. (2008). A review of results on sums of random variables. Acta Applicandae Mathematicae, 103(2), 131-140.

Ramsay, C. M. (2008). The distribution of sums of iid Pareto random variables with arbitrary shape parameter. Communications in Statistics-Theory and Methods, 37(14), 2177-2184.

Shanker, R. (2015 a): Shanker Distribution and Its Applications, International Journal of Statistics and Applications, 5 (6), 338 - 348.

Shanker, R. (2015 b): Akash Distribution and Its Applications, International Journal of Probability and Statistics, 4 (3), $65-75$.

Shanker, R. and Shukla, K.K. (2017): Ishita distribution and its Applications, Biometrics \&Biostatistics International Journal, 5(2), $1-9$. 
Shanker, R. (2017): Rani Distribution and Its Application, Biometrics \& Biostatistics International Journal, 6(1), 1 - 10 .

Shukla, K. K., \& Shanker, R. (2019). Shukla distribution and its Application. Reliability: Theory \& Applications, 14(3).

Shukla, K.K, (2018): Pranav distribution with properties and its applications, Biometrics \&Biostatistics International Journal, 7(3), $244-254$.

Shukla,K.K. (2018): Ram Awadh distribution with properties and applications, Biometrics Biostatistics International Journal, 7(6), 515 - 523.

Van Khuong, H., \& Kong, H. Y. (2006). General expression for pdf of a sum of independent exponential random variables. IEEE Communications Letters, 10(3), 159-161.

Yacoub, M. D. (2006). Simple precise approximations to Weibull sums. IEEE communications letters, 10(8), 614-616.

Zakerzadeh, H., \& Dolati, A. (2009). Generalized lindley distribution. 